\title{
An Examination of Different Explanations for the Mere Exposure Effect
}

\author{
XIANG FANG \\ SURENDRA SINGH \\ ROHINI AHLUWALIA*
}

\begin{abstract}
This article investigates two competing explanations of the mere exposure effect-the cognition-based perceptual fluency/misattribution theory (PF/M) and the affect-based hedonic fluency model (HFM) — under incidental exposure conditions. In two studies, the classical mere exposure effect is replicated in the context of banner advertising. The findings rule out the cognition-based PF/M and suggest that the spontaneous affective reaction resulting from perceptual fluency is a crucial link between fluency and evaluation. The studies provide strong evidence that the spontaneous affect influences evaluative judgments through a more complex process, likely by coloring the interpretation of the fluency experience and the nature of resulting metacognitions relating fluency to liking. Theoretical and managerial implications of the findings are mentioned.
\end{abstract}

$\mathrm{T}$ he majority of ad exposure occurs under incidental conditions-where the audiences' attention is focused elsewhere-such as reading a magazine or browsing a Web page, approximating the "mere exposure" condition, in which the target stimulus is made just accessible to the individual's perception (Zajonc 1968, 1). Repeated exposures under these conditions result in enhanced liking for a neutral stimulus.

The two major explanations for this effect are both based on the concept of "fluency," or the ease with which information is processed, but they differ in the underlying mechanisms. The perceptual fluency/misattribution model, or $\mathrm{PF} / \mathrm{M}$, utilizes a cognitive perspective and suggests that people generate inferences or metacognitions based upon their fluency experience (e.g., inferring that stimuli that come to mind more readily are better liked), thus misattributing their fluency experience to evaluations of the stimuli

\footnotetext{
*Xiang Fang is assistant professor of marketing, Spears School of Business, Oklahoma State University, Tulsa, OK 74106 (xifang@okstate.edu). Surendra Singh is Southwestern Bell Chair in Marketing, School of Business, University of Kansas, Lawrence, KS 66045 (ssingh@ @u.edu). Rohini Ahluwalia is associate professor of marketing, Carlson School of Management, University of Minnesota, Minneapolis, MN 55455 (rahluwalia@ csom.umn.edu). This article is based on the dissertation of the first author. The authors acknowledge the helpful input of the editor, associate editor, and the three reviewers. They also thank Jack Brehm, Sanjay Mishra, Dennis L. Rosen, Tracy Suter, Marlys J. Mason, and Julie Ruth for comments on earlier drafts. Corresponding author: Xiang Fang.
}

Dawn Iacobucci served as editor and Mary Frances Luce served as associate editor for this article.

Electronically published March 13, 2007
(Bornstein and D’Agostino 1992, 1994). Most marketing studies have utilized $\mathrm{PF} / \mathrm{M}$ as the explanatory mechanism (e.g., Janiszewski and Meyvis 2001; Nordhielm 2002). The hedonic fluency model, HFM (Winkielman and Cacioppo 2001), presents an alternative affect-based explanation, arguing that positive affect generated by the dynamics of fluent processing enhances stimuli evaluations (Winkielman et al. 2003). It is not clear which model (cognitive or affective) better explains mere exposure effect (MEE) under a particular set of conditions (Lee 2004; Schwarz 2004).

We test the different process explanations for the MEE in two studies using banner ads as stimuli. Since most viewers pay minimal attention to banner ads, they not only are ideal stimuli for examining the MEE but also help us accomplish a secondary goal: gaining a deeper understanding of how banner advertising works. Given the relative newness of the Web as a medium, our knowledge of how these ads influence viewers is woefully limited.

The first study examines whether the MEE is able to account for the persuasion effects of banner advertising. The second study examines the underlying process issues in the context of banner advertising.

\section{EXPLANATIONS FOR THE MERE EXPOSURE EFFECT}

Perceptual Fluency/Misattribution Model: The Cognitive Perspective

The PF/M contends that repeated exposures to a stimulus enhance its perceptual fluency. When an individual is un- 
aware of the true source (prior exposure) of the enhanced perceptual fluency, (s)he may misattribute it to liking for the stimulus or, depending upon the contextual cues provided by the task, to other relevant judgments regarding the stimulus, such as fame, truth, duration, loudness, stimulus brightness and darkness, and narrow/broad product distribution (e.g., Mandler, Nakamura, and Van Zandt 1987; Nordhielm 2002). If the individual realizes having seen the stimulus earlier, (s)he will engage in an automatic and effortless correction process, revising the initial interpretation of fluency and discounting the stimulus evaluation.

The PF/M assumes that (1) process facilitation is an affectively neutral, arousal-like experience and (2) this experience can influence extremity of the evaluation by leading to either a more positive or a more negative evaluation of the stimulus, depending on the contextual factors (Winkielman and Cacioppo 2001). Thus, if the evaluation involves darkness (brightness) judgment, more extreme evaluation of darkness (brightness) should occur.

\section{Hedonic Fluency Model: The Affective Perspective}

The HFM focuses specifically on preference judgments (as opposed to other judgments, such as brightness, fame, and truth) and argues that the dynamics of information processing itself (i.e., processing fluency) lead to positive affective responses, which in turn lead to a more positive evaluation. High fluency may generate positive affect partly because it indicates stimulus familiarity, which signals a harmless situation. It may also indicate progress toward the goal of successful recognition and coherent interpretation of the target, which is a rewarding experience (Winkielman and Cacioppo 2001).

Winkielman and Cacioppo (2001) demonstrated that perceptual fluency increases were associated with affective responses, as measured by facial electromyography (EMG). The process implied in this model is akin to the "affect-asinformation" model (Schwarz and Clore 1983), where participants infer their evaluations from how they feel (e.g., "if I feel good, I must like it"). Note that this process is also metacognitive in nature but differs from the PF/M in that the inferences are based on the affective experience and not the experience of perceptual fluency.

These data, however, are also consistent with another possibility-that affect influences the interpretation of the fluency experience and the resultant metacognitions relating fluency to liking. In some situations affect can mobilize subsequent thoughts, influencing the direction of cognitions as well as interpretation of evidence (e.g., Pham et al. 2001). Therefore, it is possible that affect may influence evaluations via its effect on interpretation of the fluency experience. More important, the EMG data also do not rule out the possibility that a parallel process, in which individuals generate metacognitions based on their fluency experience $(\mathrm{PF} / \mathrm{M})$, might also occur and influence evaluations. Therefore, these data leave the door open for an array of alternative processes by which perceptual fluency could influence evaluation.

Lee (2001, 2004) makes an insightful suggestion for testing the processes driving the effects of perceptual fluency on evaluations. She argues that if the liking responses were based on naive theories or metacognitions of the inferential nature based on the perceptual fluency experience (PF/M), then individuals would correct their judgments when they become aware of the source of perceptual fluency (e.g., noticed the repetition). However, if these responses were based on affect, then they would not be likely to exhibit this correction effect. To test this idea, Lee (2001) asked participants to count the number of triangles in abstract patterns and varied their exposure frequency (zero to six). Later they were presented with pairs and asked to make either cognitive (choosing the pattern with more triangles) or affective judgments (choosing the pattern that they liked more). Although a monotonic relationship between choice and number of exposures was obtained with affective judgments, it failed to emerge with cognitive judgments after six exposures. Lee interpreted these data as suggesting that correction processes set in for the cognitive judgments at six exposures; however, no correction occurred with affective judgments. The lack of correction effects in the affective judgment condition could possibly have emerged due to similarity between the initial task (counting the number of triangles) and the cognitive judgment (which pattern has more triangles) but not the affective judgment (which pattern is preferred). The "mismatch" of tasks in the affective judgment condition could presumably have lowered recognition likelihood in this condition. Therefore, the implications of this study for the process underlying MEE are not conclusive.

In sum, although the past decade of research has clearly established that perceptual fluency plays a key role in influencing evaluations, the jury is still out on how this effect occurs. Past literature suggests at least three potential processes: (i) fluency-based metacognitions (the cognitive route), (ii) affect-based metacognitions (affect as information), and (iii) affect influences the interpretation of the fluency experience and metacognitions generated from it. Our goals are to reconcile the evidence presented from different perspectives, revise the existing theories of the MEE, and generate a more integrative model for understanding how perceptual fluency influences evaluations.

Most prior work on MEE in marketing is based on focal exposure to target stimuli, and none has used a direct measure of perceptual fluency. The first study tests the MEE using incidental exposure to banner ads and directly measures perceptual fluency using the perceptual identification measure (e.g., Jacoby and Dallas 1981) to establish the validity of MEE in the chosen substantive context (banner advertising). The second experiment, using a misattribution paradigm, tests the different explanations based on the rationale that if individuals are made aware of the source (of either fluency or affect), they are likely to correct their evaluations. 


\section{EXPERIMENT 1: MERE EXPOSURE EFFECTS AND BANNER ADVERTISING}

\author{
Design and Procedure
}

Using a 3 (exposure frequency: zero vs. five vs. 20) $\times$ 2 (evaluation orientation: positive vs. negative responses) between-subjects design, 232 students at the University of Kansas were randomly assigned to the six cells; 13 failed to complete all parts, leaving 219 usable responses. Both positive and negative evaluation orientations were measured because generation of positive affect, associated with perceptual fluency, is expected to change the positive but not negative evaluations (Winkielman and Caccioppo 2001).

Upon arrival in a computer lab, participants were instructed to carefully read a five-page article on online education with the expectation of later answering questions about the article. The article's mood neutrality was ascertained via a pretest. Banner ads rotated (for 5 seconds each) on top of the article on the computer screen. In the fiveexposure condition, the target ad appeared once on each of the five pages, with five filler ads in the following order: $F_{1}$ $\mathrm{F}_{2} \mathrm{~T} \mathrm{~F}_{3} \mathrm{~F}_{4} \mathrm{~F}_{5}$ (where $\mathrm{T}=$ target ad and $\mathrm{F}=$ filler ad). In the 20-exposure condition, the target ad appeared four times on each page (with the same five fillers) as follows: $\mathrm{F}_{1} \mathrm{~T}$ $\mathrm{F}_{2} \mathrm{~T} \mathrm{~F}_{3} \mathrm{~T} \mathrm{~F}_{4} \mathrm{~T} \mathrm{~F}_{5}$. In the zero-exposure (control) condition, only filler ads were presented. Since participants read the article at their own pace, each page was set to appear on the screen for a minimum of 45 seconds (i.e., the respondents could not move to the next page before 45 seconds) to afford exposure opportunity for all ads (fillers and the target) on each page. After reading the article, participants responded to a few questions related to the article, followed by the key dependent measures.

\section{Stimuli}

Target Banner Ad. A full-size (468 × 60 pixels) banner ad for a digital camera with a novel and neutral brand name "Pretec" was created (pretest, $n=20: M_{\text {liking }}=$ $3.88, M_{\text {fam. }}=1.60$, seven-point scales). The ad simply featured the brand name (Pretec digital camera), pictures of digital cameras, and a "click here" button.

Layout of Web Pages. Using the E-prime 1.1 software, an interface similar to a student online magazine was developed. Banner ads were placed on the top of the article (outside of participants' focal view) to create incidental exposure, while the article on online education was placed in participants' focal view. Banner ads rotated on top, with one ad appearing at a time.

\section{Dependent Variables}

Perceptual Identification Task. A direct, objective measure of process fluency, the perceptual identification task, was administered for both the target ad and filler ads. For each banner ad, participants saw 15 visually degraded banner ad images, each appearing for 1 second. Though initially completely masked by noise, over progressive trials the ad became clearer. Participants were instructed to press any key as soon as they thought they could identify the brand name shown in the ad. Once participants pressed a key, the ad image was replaced by a question page asking them to type the brand name in a blank box. Participants were instructed to respond as quickly and as accurately as possible. The program recorded the response time (from the beginning of the presentation to the time when participants pressed the key) and brand name. Participants completed two practice trials before the actual task.

Ad Evaluation. Following Winkielman and Cacioppo (2001), participants in the positive (negative) response group were asked to rate the degree of their positive (negative) reaction to the banner ad on a nine-point scale. The scale range was one (no positive/negative reaction) to nine (very positive/negative reaction).

Recognition. In a two-alternative forced-choice recognition test, participants were shown a pair of banner ads, the target ad for Pretec and a decoy (Relisys). They were asked to select the ad that was shown on the Web pages previously.

\section{Results and Discussion}

Recognition. Consistent with the MEE, recognition scores in both treatment groups were not different from the control group (all $p$ 's $>.25$ ). The scores were not different from the .50 chance level (all $p$ 's $>.40$ ), confirming the incidental nature of the ad exposures.

Perceptual Fluency. After excluding 12\% of outliers and missing/incorrect responses (evenly distributed across conditions), a two-way ANOVA with exposure frequency and evaluative orientation as two independent variables was performed on the remaining data. A significant main effect of exposure (exp.) frequency emerged $\left(M_{0 \text { exp. }}=9,045\right.$ milliseconds, $M_{5 \text { exp. }}=8,281$ milliseconds, $M_{20 \text { exp. }}=7,895$ milliseconds; $F(2,186)=5.97, p=.003)$. Planned comparisons revealed that participants in both treatment conditions had shorter response times than those in the control condition (both $p$ 's $<.015$, one-tailed), although the means between two treatment conditions did not differ statistically $(t(186)=1.13, p<.13$, one-tailed $)$. A trend test on exposure frequency revealed a significant linear trend in response time $(F(1,189)=11.75, p=.001)$, indicating that repeated incidental exposures increase perceptual fluency of the target banner ad. See table 1.

Evaluation of the Target Banner Ad (Pretec). Oneway ANOVAs were conducted on both positive and negative evaluations of the target banner ad (Pretec). Consistent with past research, there was a significant main effect of exposure frequency in the positive evaluation condition $\left(M_{0 \text { exp. }}=\right.$ $3.91, \quad M_{5 \text { exp. }}=4.73, \quad M_{20 \text { exp. }}=5.11 ; \quad F(2,104)=3.24$, $p<.05)$. Participants in both treatment groups evaluated the 
TABLE 1

MEANS AND STANDARD DEVIATIONS OF DEPENDENT VARIABLES IN STUDY 1

\begin{tabular}{|c|c|c|c|c|c|c|}
\hline & \multicolumn{3}{|c|}{ Positive evaluation } & \multicolumn{3}{|c|}{ Negative evaluation } \\
\hline & $\begin{array}{l}0 \text { exp. } \\
(n=34)\end{array}$ & $\begin{array}{l}5 \text { exp. } \\
(n=37)\end{array}$ & $\begin{array}{l}20 \text { exp. } \\
(n=36)\end{array}$ & $\begin{array}{l}0 \text { exp. } \\
(n=39)\end{array}$ & $\begin{array}{l}5 \text { exp. } \\
(n=37)\end{array}$ & $\begin{array}{l}20 \text { exp. } \\
(n=36)\end{array}$ \\
\hline Perceptual fluency ( $n=$ sample size $)^{\mathrm{a}}$ & $\begin{array}{r}8,960 \\
(1,768)\end{array}$ & $\begin{array}{r}8,246 \\
(1,666)\end{array}$ & $\begin{array}{r}7,856 \\
(1,711)\end{array}$ & $\begin{array}{r}9,118 \\
(2,257)\end{array}$ & $\begin{array}{r}8,320 \\
(1,793)\end{array}$ & $\begin{array}{r}7,936 \\
(2,112)\end{array}$ \\
\hline$n$ & 31 & 34 & 31 & 36 & 31 & 29 \\
\hline$A_{a d}$ & $\begin{array}{c}3.91 \\
(2.08)\end{array}$ & $\begin{array}{c}4.73 \\
(2.04)\end{array}$ & $\begin{array}{c}5.11 \\
(1.91)\end{array}$ & $\begin{array}{c}3.31 \\
(1.85)\end{array}$ & $\begin{array}{c}3.05 \\
(2.24)\end{array}$ & $\begin{array}{c}3.39 \\
(2.02)\end{array}$ \\
\hline Recognition & .53 & .51 & .56 & .46 & .57 & .58 \\
\hline
\end{tabular}

NOTE.- exp. = exposures.

aThe smaller sample size in each group is due to missing responses, incorrect responses, and outliers.

target ad more positively than those in the control group (both $p$ 's $<.05$, one-tailed), although there was no difference between the two treatment groups $(t<1)$. In contrast, a significant main effect of exposure frequency did not emerge in the negative evaluation condition $\left(M_{0 \text { exp. }}=3.31, M_{5 \text { exp. }}=\right.$ $\left.3.05, M_{20 \text { exp. }}=3.39 ; F<1\right)$. A trend test revealed a significant linear trend in positive evaluations as exposure frequency increased $(F(1,104)=6.23, p<.015)$ but not with negative evaluations $(F<1)$.

Discussion. The pattern of obtained results indicates that repeated incidental exposures to banner ads resulted in increased perceptual fluency without increasing recognition. Consistent with past research, we found increased perceptual fluency to be accompanied with more positive evaluations of the ad but not with negative evaluations, suggestive of a positive affect. Note, however, that the perceptual identification task used in this study is an "objective" measure of perceptual fluency that provides direct evidence of fluency with repeated exposures to stimuli (supporting the MEE). Assessments of subjective fluency (the conscious experience of processing ease that may become disassociated from objective assessments of fluency; Winkielman et al. 2003, 193), though, are needed to clearly distinguish between the different explanations and to test mediation, since these mechanisms are based on metacognitions resulting from the subjective experience of fluency. (Interestingly, a mediation analysis using the "objective" measure from this study did not support a significant mediation of exposure frequency on evaluation by fluency. Therefore, subjective fluency is measured in study 2.)

\section{EXPERIMENT 2: MISATTRIBUTION EFFECTS}

\section{Objective and Predictions}

The underlying logic of this experiment is that people use an input (affect or cognition) for judgments only as long as it is informative (Schwarz and Clore 1983), or else they tend to discount its effects on judgments. If participants misattribute the basis of their evaluations (fluency or affect) to another source (e.g., music), then they would correct their judgments.

We provided participants with the opportunity to misattribute either their fluency experience or their felt affect to another source (background music). If consistent with $\mathrm{PF} / \mathrm{M}$, participants' liking judgments are solely based on the fluency experience and its resultant metacognitions; correction effects would emerge only in the fluency-misattribution condition. However, if their evaluations are driven by the "affect-as-information" model, they would likely exhibit a correction effect in the affect-misattribution condition (affect attributed to the music and, therefore, no longer informative about the ad) but not in the fluency-misattribution condition, as their evaluation is based on the generated affect and not the fluency experience per se (see Lee [2001] for a similar argument). However, if the generated affect plays a more complex role by influencing the participant's interpretation of the fluency experience and mobilization of related thoughts, then the ad evaluations would be corrected in both misattribution conditions, since both affect and the fluency experience are involved in an interrelated manner in these metacognitions. Lee (2004) argues that the more spontaneous and automatic the affect (e.g., affect as information), the less likely it is to undergo correction; however, the higher the likelihood of cognitions, the greater the possibility of correction. If Lee's argument holds, then correction effects under the affect-misattribution condition would be less likely if participants were using affect solely as information and not utilizing it to interpret the fluency experience.

\section{Design and Participants}

In a 2 (exposure frequency: zero vs. five) $\times 4$ (misattribution: fluency vs. affect vs. general caution vs. no attribution) design, 304 undergraduate students were randomly assigned to eight cells. Misattribution manipulation was adapted from Fazendeiro et al. (2005). In the fluency-misattribution group, participants were told that the background music might make it easier to process the information they were exposed to, which might give them a sense of fluency (things coming to their mind faster or easier). They were asked to make the next set of evaluations ignoring the feeling 
TABLE 2

MEANS AND STANDARD DEVIATIONS OF DEPENDENT VARIABLES IN STUDY 2

\begin{tabular}{|c|c|c|c|c|c|c|c|c|}
\hline & \multicolumn{8}{|c|}{ Misattribution instruction } \\
\hline & \multicolumn{2}{|c|}{ No instruction } & \multicolumn{2}{|c|}{ General caution } & \multicolumn{2}{|c|}{ Fluency } & \multicolumn{2}{|c|}{ Affect } \\
\hline & $\begin{array}{c}0 \text { exp. } \\
(n=37)\end{array}$ & $\begin{array}{c}5 \text { exp. } \\
(n=40)\end{array}$ & $\begin{array}{c}0 \text { exp. } \\
(n=38)\end{array}$ & $\begin{array}{c}5 \text { exp. } \\
(n=36)\end{array}$ & $\begin{array}{c}0 \text { exp. } \\
(n=39)\end{array}$ & $\begin{array}{c}5 \text { exp. } \\
(n=38)\end{array}$ & $\begin{array}{c}0 \text { exp. } \\
(n=39)\end{array}$ & $\begin{array}{c}5 \text { exp. } \\
(n=37)\end{array}$ \\
\hline$A_{a d}$ & $\begin{array}{c}4.75 \\
(1.59)\end{array}$ & $\begin{array}{c}5.92 \\
(1.90)\end{array}$ & $\begin{array}{c}4.76 \\
(1.47)\end{array}$ & $\begin{array}{l}5.77 \\
(2.03)\end{array}$ & $\begin{array}{c}4.81 \\
(1.22)\end{array}$ & $\begin{array}{c}4.72 \\
(1.86)\end{array}$ & $\begin{array}{c}4.83 \\
(1.72)\end{array}$ & $\begin{array}{c}4.74 \\
(1.82)\end{array}$ \\
\hline Ad-induced feeling & $\begin{array}{c}3.77 \\
(1.86)\end{array}$ & $\begin{array}{c}4.46 \\
(1.67)\end{array}$ & $\begin{array}{c}3.52 \\
(1.45)\end{array}$ & $\begin{array}{c}4.39 \\
(1.76)\end{array}$ & $\begin{array}{c}3.58 \\
(1.59)\end{array}$ & $\begin{array}{c}3.33 \\
(1.64)\end{array}$ & $\begin{array}{c}4.01 \\
(1.48)\end{array}$ & $\begin{array}{c}3.61 \\
(1.43)\end{array}$ \\
\hline Subjective fluency & $\begin{array}{c}3.22 \\
(2.24)\end{array}$ & $\begin{array}{l}3.98 \\
(2.39)\end{array}$ & $\begin{array}{l}2.63 \\
(2.02)\end{array}$ & $\begin{array}{c}4.11 \\
(2.69)\end{array}$ & $\begin{array}{l}3.10 \\
(2.07)\end{array}$ & $\begin{array}{l}2.82 \\
(2.14)\end{array}$ & $\begin{array}{c}4.00 \\
(2.49)\end{array}$ & $\begin{array}{l}3.62 \\
(2.52)\end{array}$ \\
\hline Recognition & .51 & .48 & .50 & .53 & .54 & .55 & .56 & .51 \\
\hline
\end{tabular}

NOTE.-exp. $=$ exposures.

of fluency. In the affect-misattribution group, participants were told that the music might induce different types of feelings. They were instructed to make the evaluations ignoring their feelings. In addition, a general-caution condition was included to help assess whether any caution, irrespective of the focus on affect or fluency, might reduce evaluations. In this condition, participants were told that the background music might influence their judgments and were instructed to make the evaluations ignoring the effects of background music. No music-related instructions were given in the control condition.

\section{Procedure and Measures}

Upon arrival in the computer lab, participants were instructed to carefully read the same five-page article on online education from study 1 and to expect to answer several questions about the article. Exposure frequency was manipulated as in study 1 . During the entire study 2, participants were exposed, through headphones, to a piece of soft meditation music. ${ }^{1}$ After reading the article, participants answered a few questions about it. Before making their evaluative judgments, they received different misattribution instructions as discussed above.

Attitude toward the ad was measured with a traditional bipolar attitude measure (three nine-point items: dislike/like, unpleasant/pleasant, and bad/good; Cronbach's alpha = .93). To assess ad-induced feelings, following Madden, Allen, and Twible (1988), participants responded to the prompt, "How does this ad make you feel?" followed by seven adjectives (good, pleased, cheerful, joyous, amused, playful, and stimulated; Cronbach's alpha $=.94$ ) on ninepoint scales (not at all/very much so). Subjective fluency was assessed by asking participants to indicate how easy it was for them to process the ad (nine-point scale: very easy to process/very difficult to process). Finally, participants responded to manipulation check questions (described subsequently), a recognition test, and demographic items.

${ }^{1}$ The music can be requested from the authors.

\section{Results and Discussion}

Manipulation Checks. More than $80 \%$ of respondents in each condition responded positively to a general manipulation check question ("Did you take the information in instructions into account when making your judgments?") adopted from Fazendeiro et al. (2005). Responses to the two specific questions relating to misattributions (extent to which they ignored the effects of [i] ease of processing the stimuli and [ii] feelings aroused on nine-point scales) are discussed next. Participants in the fluency-misattribution condition $\left(M_{\text {flu. }}=5.60\right)$ were more likely to report ignoring the fluency effects than those in other instruction conditions $\left(M_{\text {no ins. }}=\right.$ $4.51, M_{\text {gen. cau. }}=4.96$, and $M_{\text {affect }}=4.96$; all $p$ 's $<.02$, onetailed). Similarly, respondents in the affect-misattribution condition $\left(M_{\text {affect }}=5.47\right)$ were more likely to ignore the effects of feelings aroused than those in other conditions $\left(M_{\text {no ins. }}=\right.$ $4.69, t(300)=2.17, p<.02 ; M_{\text {gen. cau. }}=5.18, t(300)<1$; and $M_{\text {flu. }}=4.92, t(300)=1.52, p<.07$; all one-tailed). These results indicate that participants followed the instructions when making their evaluations. Recognition scores in all groups did not differ from the .50 chance level (all $p$ 's $>.40)$, verifying the incidental nature of ad exposure. See table 2.

Attitude toward the Target Banner Ad. A two-way ANOVA on attitude toward the ad revealed a significant interaction effect of exposure frequency and misattribution $(F(3,296)=3.02, p=.03)$. Participants in the five-exposure condition evaluated the ad more positively than those in the control in both the no-misattribution $\left(M_{0 \text { exp. }}=4.75\right.$, $M_{5 \text { exp. }}=5.92 ; F(1,296)=8.91, p<.002$, one-tailed) and general-caution conditions $\left(M_{0 \text { exp. }}=4.76, M_{5 \text { exp. }}=5.77\right.$; $F(1,296)=6.34, p<.01$, one-tailed). Correction effects, however, emerged in both the fluency- and the affect-misattribution conditions, so that the difference between the control and five-exposure conditions was not significant (fluency misattribution: $M_{0 \text { exp. }}=4.81, M_{5 \text { exp. }}=4.72$; affect misattribution: $M_{0 \text { exp. }}=4.83, M_{5 \text { exp. }}=4.74$; both $F$ 's $<1$ ). By demonstrating that misattribution of either the fluency 
signal or the affective experience eliminates the fluency's effect on judgments, these results clearly support the more complex model in which affect influences the interpretation of fluency.

Ad-Induced Feelings. A marginally significant main effect of misattribution $(F(3,296)=2.33, p<.08)$ qualified by a significant interaction of exposure frequency and misattribution $(F(3,296)=3.04, p<.03)$ emerged. Planned comparisons revealed that in both the no-misattribution and general-caution conditions, participants in the five-exposure condition reported more positive feelings than those in the control group (no misattribution: $M_{0 \text { exp. }}=3.77, M_{5 \text { exp. }}=$ 4.46; $F(1,296)=3.53, p<.035$, one-tailed; general caution: $M_{0 \text { exp. }}=3.52, M_{5 \text { exp. }}=4.39 ; F(1,296)=5.42, p<.02$, one-tailed). Significant differences in the ad-induced feelings between the five-exposure and control groups did not emerge in the fluency- $\left(M_{0 \text { exp. }}=3.58, M_{5 \exp .}=3.33 ; F<1\right)$ and the affect-misattribution conditions $\left(M_{0 \text { exp. }}=4.01, M_{5 \text { exp. }}=\right.$ $3.61 ; F(1,296)=1.22, p>.13$, one-tailed), where subjects exhibited correction effects.

Subjective Fluency. A two-way ANOVA on the subjective fluency measure revealed a significant interaction effect of exposure frequency and misattribution $(F(3,296)=$ $2.74, p<.05$ ). Planned comparisons revealed that participants in the five-exposure group perceived the ad easier to process than those in the control group, in both the no-misattribution (marginally significant: $M_{0 \text { exp. }}=3.22$, $M_{5 \exp .}=3.98 ; F(1,296)=2.04, p<.08$, one-tailed) and the general-caution conditions $\left(M_{0 \text { exp. }}=2.63, M_{5 \text { exp. }}=\right.$ $4.11 ; F(1,296)=7.46, p<.005$, one-tailed). No significant differences in subjective fluency were found between the five-exposure and the control groups in both the fluency- $\left(M_{0 \text { exp. }}=3.10, M_{5 \exp .}=2.82 ; F<1\right)$ and the affect-misattribution conditions $\left(M_{0 \text { exp. }}=4.00, M_{5 \text { exp. }}=\right.$ $3.62 ; F<1)^{2}$

Discussion. The results of experiment 2 clearly rule out a purely cognitive explanation of fluency's effects on evaluations and suggest that not only does the fluency experience generate affect but that the generated affect is also likely to influence subsequent cognitions as well as interpretation of the fluency experience.

\section{GENERAL DISCUSSION}

We conducted two studies to understand the processes underlying MEE in incidental ad exposure conditions. In study 1, the classical MEE was replicated in the domain of banner ads: repeated incidental exposures to banner ads, just under the level of perception, increased perceptual fluency and resulted in more positive evaluations. In study 2 , we

\footnotetext{
${ }^{2}$ A series of mediation analyses (based on Baron and Kenny 1986) suggest that both subjective fluency and ad-induced feelings mediate the effect of exposure frequency and misattribution on $\mathrm{A}_{\mathrm{ad}}$. In addition, the ad-induced feelings partially mediate the effect of subjective fluency on $\mathrm{A}_{\mathrm{ad}}$. In other words, fluency experience and the resulting affect provide a crucial link between exposure frequency and ad evaluations.
}

used the misattribution paradigm to test the underlying processes and found that misattribution of either the fluency signal or the affective experience eliminated the MEE, which supported the more complex process whereby the fluency experience generates affect and the generated affect influences subsequent cognitions and the interpretation of the fluency experience.

Although our studies focus on affective (preference) judgments, our findings may also enhance understanding of cognitive judgments (e.g., truth, fame, and brightness). When exposure to a stimulus occurs under conditions of mere exposure, respondents are likely to have at least two available inputs at the time of judgment: fluency experience and affect. If affect is perceived to be diagnostic (as with preference judgments), it is likely to be used as an input in decision making (affect as information) and may be instrumental in interpreting other inputs (e.g., the fluency experience). However, when affect is not diagnostic for the judgment at hand (e.g., fame, brightness, and truth), it is not likely to be used as an input in decision making (e.g., Feldman and Lynch 1988). Instead, individuals might generate other types of naive theories or metacognitions (e.g., easy to retrieve, so must be brighter) to interpret the fluency experience. Importantly, these effects are likely to appear only when no other diagnostic information is available, and the judgment is based on whatever little information is available in the evaluation setting. One caveat: our findings may be limited to low-involvement, incidental ad processing conditions.

One practical implication of this research is that online advertisers might be placing excessive emphasis on the click-through rates-the primary metric for measuring the effectiveness of online ads. Our results suggest that even when there is no overt sign of effectiveness, such as recognition or click through, the banner ads may still impact ad liking.

Past research suggests that reliance on affect tends to increase when cognitive resources are severely constrained (e.g., Shiv and Fedorikhin 1999), given the more automatic and less resource-intensive nature of affective processes. Our finding that affect is a key process underlying the mere exposure effect implies that these effects are expected to strengthen under resource-constrained decision-making situations (e.g., limited cognitive capacity, lack of motivation to process information, and time pressure), which are prevalent in marketplace environments.

Additionally, findings from our study 1 suggest that consumers tend to have a relatively high level of tolerance for repeated exposure to banner ads-the wear-out effects of banner ads did not kick in even after 20 exposures in this experiment. A future research issue might involve examining the exposure levels at which wear-out effects are likely to emerge. Another issue that would benefit from future research is the generalizability of these effects to brand evaluations.

Repetition is just one of many variables that may influence fluency and hence evaluations. Variables, such as symmetry, 
harmony, and complexity, that govern aesthetic experience also affect fluency experience (Schwarz 2004). Therefore, our results may be tangentially relevant to logo design, point-ofpurchase display, or product design-domains where aesthetics play an important role.

\section{REFERENCES}

Baron, Reuben M. and David A. Kenny (1986), "The ModeratorMediator Variable Distinction in Social Psychological Research: Conceptual, Strategic, and Statistical Considerations," Journal of Personality and Social Psychology, 31 (December), 1173-82.

Bornstein, F. Robert and Paul R. D’Agostino (1992), "Stimulus Recognition and the Mere Exposure Effect," Journal of Personality and Social Psychology, 63 (October), 545-52.

- (1994), "The Attribution and Discounting of Perceptual Fluency: Preliminary Tests of a Perceptual Fluency/Attributional Model of the Mere Exposure Effect," Social Cognition, 12 (Summer), 103-28.

Fazendeiro, Tedra, Piotr Winkielman, Chun Luo, and Christopher Lorah (2005), "False Recognition across Meaning, Language, and Stimulus Format: Conceptual Relatedness and the Feeling of Familiarity," Memory and Cognition, 33 (2), 249-60.

Feldman, Jack M. and John G. Lynch Jr. (1988), "Self-Generated Validity and Other Effects of Measurement on Belief, Attitude, Intention and Behavior," Journal of Applied Psychology, 73 (August), 421-35.

Jacoby, Larry L. and Mark Dallas (1981), "On the Relationship between Autobiographical Memory and Perceptual Learning," Journal of Experimental Psychology: General, 110 (3), 306-40.

Janiszewski, Chris and Tom Meyvis (2001), "Effects of Brand Logo Complexity, Repetition, and Spacing on Processing Fluency and Judgment," Journal of Consumer Research, 28 (June), 18-32.

Lee, Angela Y. (2001), “The Mere Exposure Effect: An Uncertainty Reduction Explanation Revisited," Personality and Social Psychology Bulletin, 27 (10), 1255-66.
(2004), "The Prevalence of Metacognitive Routes to Judgment," Journal of Consumer Psychology, 14 (4), 349-55.

Madden, Thomas J., Chris T. Allen, and Jacquelyn L. Twible (1988), "Attitude toward the Ad: An Assessment of Diverse Measurement Indices under Different Processing 'Sets,', Journal of Marketing Research, 25 (3), 242-52.

Mandler, George, Yoshio Nakamura, and Billie J. Van Zandt (1987), "Nonspecific Effects of Exposure on Stimuli That Cannot Be Recognized," Journal of Experimental Psychology: Learning, Memory, and Cognition, 13 (4), 646-48.

Nordhielm, Christie L. (2002), "The Influence of Level of Processing on Advertising Repetition Effects," Journal of Consumer Research, 29 (December), 371-82.

Pham, Michel Tuan, Joel B. Cohen, John W. Pracejus, and David G. Hughes (2001), "Affect Monitoring and the Primacy of Feelings in Judgment," Journal of Consumer Research, 28 (September), 167-88.

Schwarz, Norbert (2004), "Metacognitive Experiences in Consumer Judgment and Decision Making," Journal of Consumer Psychology, 14 (4), 332-48.

Schwarz, Norbert and Gerald L. Clore (1983), "Mood, Misattribution, and Judgments of Well-Being: Informative and Directive Functions of Affective States," Journal of Personality and Social Psychology, 45 (3), 513-23.

Shiv, Baba and Alexander Fedorikhin (1999), "Heart and Mind in Conflict: The Interplay of Affect and Cognition in Consumer Decision Making," Journal of Consumer Research, 26 (December), 278-92.

Winkielman, Piotr and John T. Cacioppo (2001), "Mind at Ease Puts a Smile on the Face: Psychophysiological Evidence That Processing Facilitation Increases Positive Affect," Journal of Personality and Social Psychology, 81 (6), 989-1000.

Winkielman, Piotr, Norbert Schwarz, Tedra A. Fazendeiro, and Rolf Reber (2003), "The Hedonic Marking of Processing Fluency: Implications for Evaluative Judgment," in The Psychology of Evaluation: Affective Processes in Cognition and Emotion, ed. Jochen Musch and Karl C. Klauer, Mahwah, NJ: Erlbaum, 189-217.

Zajonc, Robert (1968), "Attitudinal Effects of Mere Exposure," Journal of Personality and Social Psychology Monographs, 9 (No. 2, Part 2), 1-27. 